\title{
Structural phase transitions and equations of state for selenium under pressure
}

\author{
G. Parthasarathy* and W. B. Holzapfel \\ Universität Gesamthochschule, Paderborn, Fachbereich Physik, D-4790 Paderborn, Federal Republic of Germany
}

(Received 24 June 1988)

\begin{abstract}
Pressure-induced structural phase transitions in hexagonal Se were studied for the first time by high-pressure energy dispersive $\mathrm{x}$-ray-diffraction techniques up to $50 \mathrm{GPa}$. The observed phase transitions are as follows: from hexagonal to monoclinic at $14(1) \mathrm{GPa}$, from monoclinic to tetragonal at $28(2) \mathrm{GPa}$, and from tetragonal to rhombohedral at 41(3) $\mathrm{GPa}$. All the observed phase transitions are reversible with hysteresis. The high-pressure behavior of selenium is compared with that of tellurium. The room-temperature equation-of-state data for all the phases are presented.
\end{abstract}

Selenium and tellurium are known to exhibit several interesting physical phenomena, ${ }^{1}$ one of which is the pressure-induced semiconductor-metal transition. ${ }^{2-5}$ The high-pressure metallic phases are superconducting at sufficiently low temperatures. ${ }^{6-8}$ There has been also considerable interest in theoretical understanding of the electronic structure of $\mathrm{Se}$ and $\mathrm{Te}$ at high pressures. ${ }^{9-15}$ However, so far all the theoretical work ${ }^{9-15}$ on Se and Te is limited to pressures below $14 \mathrm{GPa}$, due to the lack of structural data for these materials in the extended pressure range.

In a recent communication, ${ }^{16}$ we have reported that $\mathrm{Te}$ undergoes many structural phase transitions under high pressure. Since the elements in many groups of the periodic system exhibit a close homology in their highpressure behavior, ${ }^{17-20}$ one could anticipate that $\mathrm{Se}$ might exhibit as similar a sequence of structural phase transition as Te.

In a high-pressure $\mathrm{x}$-ray investigation McCann and Cartz $^{21}$ found that hexagonal and amorphous Se transform irreversibly at 14(1) $\mathrm{GPa}$ to similar unidentified structures. On the contrary, Moodenbaugh, $\mathrm{Wu}$, and Viswanathan ${ }^{7}$ have reported that the pressure-induced transition in hexagonal $\mathrm{Se}$ is reversible. In a recent study, ${ }^{22}$ it has been reported that Se undergoes several pressure-induced transitions up to $50 \mathrm{GPa}$. In that study ${ }^{22}$ the observed interplanar spacings $d_{h k l}$ at $4.5 \mathrm{GPa}$ (in particular strong diffraction lines at $2.286 \AA, 2.606 \AA$ ) indicate that the starting material was already a phase mixture. It is well known for Se that a sample with phase mixture yields differing results. ${ }^{23,24}$ However, in none of the above studies, have structures of high-pressure phases been solved.

These facts have motivated the present study on the room-temperature phase diagram of hexagonal Se up to $50 \mathrm{GPa}$.

In the present Rapid Communication, we report results of energy dispersive $x$-ray diffraction on hexagonal selenium under pressure at room temperature. We find that hexagonal Se I transforms to a monoclinic phase Se II at 14(1) GPA, Se II transforms to a tetragonal phase Se III at 28(2) $\mathrm{GPa}$, and Se III transforms to a rhombohedral phase Se IV at 41 (3) GPa. All the observed phase transitions are reversible with hysteresis. We also present the room- temperature equation-of-state data for all the observed phases of Se, which hopefully helps theorists to evaluate the structural stability of Se at high pressures.

The powdered samples of Se with $99.999 \%$ purity yielded at room temperature and ambient pressure an $x$-raydiffraction pattern of the pure hexagonal phase with $a=4.366$ (1) $\AA$ and $c=4.958(2) \AA$ in excellent agreement with the literature values ${ }^{25} a=4.3655(10) \AA$ and $c=4.9576(24) \AA$. The diamond-anvil technique ${ }^{26,27}$ was used for generating high pressure and the well-known ruby fluorescence technique $\mathrm{e}^{28-31}$ served for pressure measurement. Liquid nitrogen was used as a pressure transmitting medium in three of the present experiments and a mixture of methanol:ethanol:water in the ratio 16:3:1 was applied in the other two experiments to check on possible effects of nonhydrostatic stresses. ${ }^{32}$ All the five different experiments yielded consistent results. The hysteresis of the phase transitions was studied by taking data not only on increasing but also on decreasing pressure. The values of the transition pressures quoted in the paper correspond, therefore, within the given uncertainties to the equilibrium transition pressures. The precision in the pressure determination with respect to the nonlinear ruby scale ${ }^{33}$ is estimated to be better than $0.5 \mathrm{GPa}$ at 40 $\mathrm{GPa}$.

The structural data were obtained by energy dispersive $\mathrm{x}$-ray diffraction. The details of the experimental technique have been described elsewhere. ${ }^{34-36}$

The variation of the observed lattice spacings $d_{h k l}$ of Se as a function of pressure are represented in Fig. 1. The appearance and disappearance of the diffraction lines illustrates clearly the structural phase transitions in Se under high pressure. It is also seen from Fig. 1 that all the observed phase transitions are reversible with hysteresis. The indexing $(h k l)$ of the observed lattice spacings is presented in Table I for the different phases together with the observed and fitted $d_{h k l}$ values. The variation of the lattice spacings for hexagonal $\mathrm{Se} I$ corresponds closely to the data reported earlier in the literature. ${ }^{37,38}$ The values of the lattice parameters at $15 \mathrm{GPa}[a=3.640$ (1) $\AA$ and $c=5.160(2) \AA]$ indicate that the axial ratio $c / a$ of $\mathrm{Se} I$ increases from $1.135(1)$ at ambient pressure to $1.417(1)$ at $15 \mathrm{GPa}$. The observed variation of $c / a$ with pressure is consistent with the published data. ${ }^{34,35} \mathrm{~A}$ pressure- 


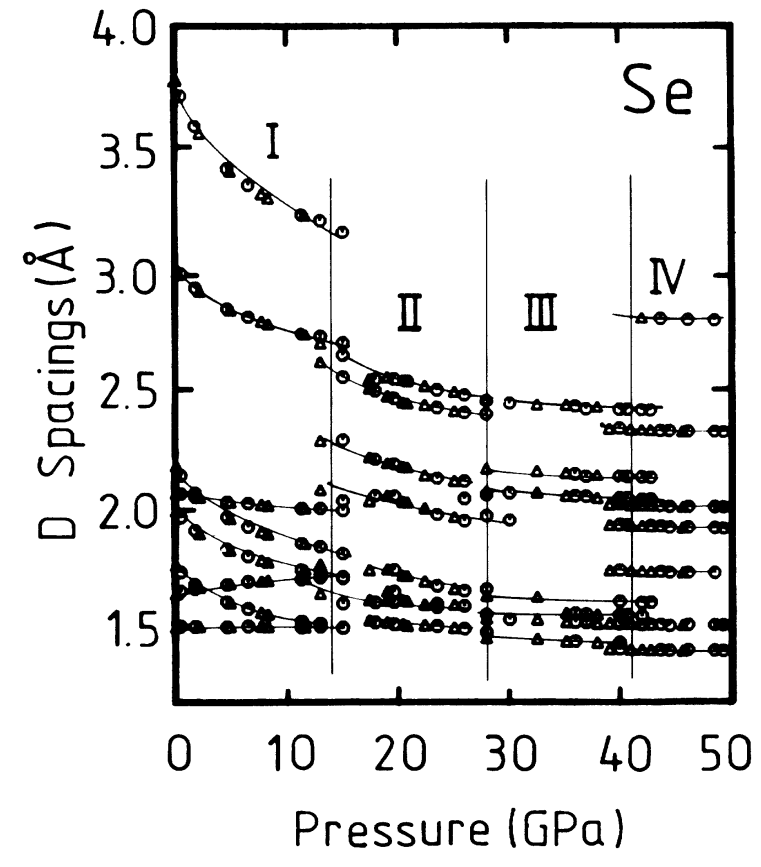

FIG. 1. Effect of pressure on $d$ spacings for various phases of selenium. Circles and triangles indicate data collected on increasing and decreasing pressure, respectively. The lines drawn through the experimental data points are only guides for the eye. induced phase transition occurs in hexagonal $\mathrm{Se}$ at a $c / a$ ratio of 1.417 , which corresponds to the value of the $c / a$ ratio of Te at 4.0 (2) $\mathrm{GPa}$ (Ref. 16) where it undergoes its first phase transition. Since the initial $c / a$ ratios for Se and $\mathrm{Te}$ are 1.13 and 1.33 , respectively, one could already expect from simple scaling rules that $\mathrm{Se}$ would require a higher pressure for this transformation to occur. The data for Se II could be assigned only to a monoclinic structure apparently similar to the structure of Te II phase, ${ }^{39}$ however, with three atoms per unit cell. Since the intensities of the observed reflections may be affected by the preferred orientations in the sample a complete structure determination cannot be made with these data; however, the values of the unit-cell parameters (Table I) and the different numbers of atoms in the unit cell indicate clearly that $\mathrm{Se}$ II has a different structure than Te II.

It is quite evident from Fig. 1 that Se II transforms to Se III at 28(2) GPa. On the basis of the known highpressure sequence of Te (Ref. 16) one may be tempted to assign an orthorhombic structure to Se III; however, the observed pattern can be indexed unambiguously only to a tetragonal structure with four atoms per unit cell. The observed and calculated interatomic spacings for Se III at 40(2) $\mathrm{GPa}$ are also listed in Table I.

In Fig. 1, new diffraction lines at 41(3) GPa illustrate the appearance of a new phase Se IV. The diffraction pattern for SeIV can be indexed as rhombohedral with two

TABLE I. Selected structural data for each of the high-pressure phases of selenium including atomic volume $V$, lattice parameters $a, b, c$, and $\beta$ (where adequate), as well as indexing $(h k l)$ for the experimental lattice spacings $d_{\text {obs }}$ and the best-fit values $d_{\text {calc. }}$

\begin{tabular}{|c|c|c|c|c|c|}
\hline \multirow[b]{2}{*}{$(h k l)$} & \multicolumn{2}{|c|}{$\begin{array}{c}\mathrm{Se} \mathrm{I} \\
P=4.6(2) \mathrm{GPa}, V=22.67(14) \AA^{3} \\
a=3.939(1) \AA, c=5.062 \AA\end{array}$} & \multicolumn{3}{|c|}{$\begin{array}{c}\text { Se II } \\
P=19.0(9) \mathrm{GPa}, V=14.96(8) \AA^{3} \\
a=3.2712(7) \AA, b=4.1264(8) \AA \\
c=3.3484(9) \AA, \beta=96.80(2)^{\circ}\end{array}$} \\
\hline & $d_{\text {obs }}$ & $d_{\text {calc }}$ & $(h k l)$ & $d_{\text {obs }}$ & $d_{\text {calc }}$ \\
\hline 10.0 & $3.412(3)$ & 3.411 & 110 & $2.548(3)$ & 2.552 \\
\hline 10.1 & $2.828(2)$ & 2.829 & $10 \overline{1}$ & $2.468(3)$ & 2.474 \\
\hline 10.2 & $2.034(2)$ & 2.033 & 101 & $2.200(3)$ & 2.197 \\
\hline 11.0 & $1.969(1)$ & 1.970 & 020 & $2.062(2)$ & 2.063 \\
\hline 11.1 & $1.835(2)$ & 1.836 & 021 & $1.756(2)$ & 1.753 \\
\hline 00.3 & $1.687(1)$ & 1.687 & 002 & $1.661(2)$ & 1.662 \\
\hline 20.1 & $1.617(1)$ & 1.616 & 200 & $1.620(3)$ & 1.624 \\
\hline \multirow[t]{2}{*}{10.3} & $1.512(2)$ & 1.512 & $20 \overline{1}$ & $1.540(4)$ & 1.533 \\
\hline & \multicolumn{2}{|c|}{$\begin{array}{c}\text { Se III } \\
P=40(2) \mathrm{GPa}, V=12.71(3) \AA^{3} \\
a=4.585(2) \AA, c=2.418(1) \AA\end{array}$} & \multicolumn{3}{|c|}{$\begin{array}{c}\mathrm{Se} \mathrm{IV} \\
P=46.2(23) \mathrm{GPa}, V=12.08(1) \AA^{3} \\
a=4.658(2) \AA, c=3.858(2) \AA\end{array}$} \\
\hline$(h k l)$ & $d_{\text {obs }}$ & $d_{\text {calc }}$ & $(h k l)$ & $d_{\text {obs }}$ & $d_{\text {calc }}$ \\
\hline 001 & $2.418(2)$ & 2.418 & 10.1 & $2.788(2)$ & 2.788 \\
\hline 101 & $2.140(1)$ & 2.139 & 11.0 & $2.328(2)$ & 2.329 \\
\hline 210 & $2.051(1)$ & 2.051 & 20.0 & $2.016(2)$ & 2.017 \\
\hline 111 & $1.938(2)$ & 1.939 & 00.2 & $1.929(2)$ & 1.929 \\
\hline 220 & $1.621(1)$ & 1.621 & 10.2 & $1.740(1)$ & 1.740 \\
\hline 211 & $1.564(1)$ & 1.564 & 21.0 & $1.524(1)$ & 1.525 \\
\hline 300 & $1.528(2)$ & 1.528 & 21.1 & $1.420(1)$ & 1.418 \\
\hline 310 & $1.450(2)$ & 1.450 & & & \\
\hline
\end{tabular}




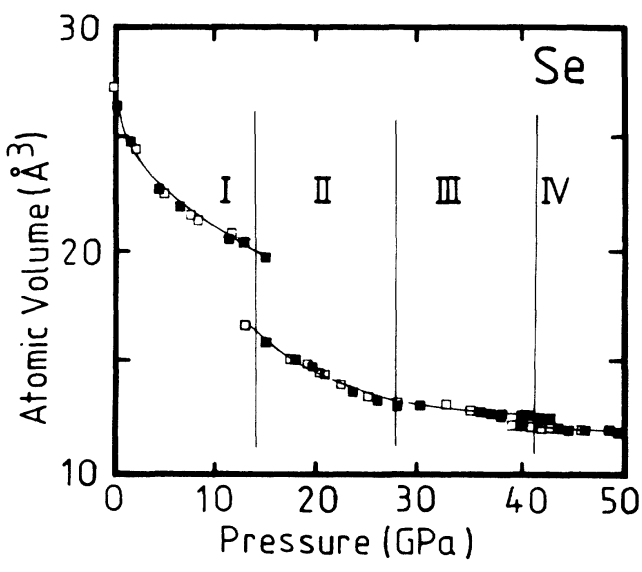

FIG. 2. Effect of pressure on the atomic volume of selenium in the different phases. The closed and open squares indicate data collected on increasing and decreasing pressure, respectively. The continuous lines represent equations of state obtained by least-squares fits. The fit parameters are given in Table II.

atoms per unit cell. The lattice spacings for Se IV at 46.2(23) GPa (Table I) yield the lattice parameters $a=2.981(2) \AA$ and $\alpha=102.76(12)^{\circ}$ for the rhombohedral cell and $a=4.6581(12) \AA$ and $c=3.858(2) \AA$ for the equivalent hexagonal cell.

From a comparison with the high-pressure behavior of Te (Ref. 16) one might expect that Se IV would ultimately also transform to a body-centered-cubic structure $\mathrm{Se} v$, when $c / a$ approaches a value $c / a=0.812$. However, within the pressure range $39-49 \mathrm{GPa}, c / a=0.828(2)$ of Se IV remains constant within the experimental uncertainty. This observation seems to indicate that the possible Se IV-SeV transition may require much higher pressure than the upper limit of the present experimental investigation.

The variation of the atomic volume for all the presently studied phases of Se under pressure at room temperature are represented in Fig. 2 which shows clearly that the transitions SeI-Se II and Se III-SeIV are first-order phase transitions with volume discontinuities of 20 and 3.5\%, respectively. On the other hand, the volume change at the Se II-Se III phase transition appears to be smaller than $1 \%$. The curves in Fig. 2 represent fits of first-order Murnaghan equations

$$
\left(V / V_{r}\right)=\left[\left(B_{r}^{\prime} / B_{r}\right)\left(P-P_{r}\right)+1\right]^{-1 / B_{r}^{\prime}}
$$

where free parameters $V_{r}, B_{r}$, and $B_{r}^{\prime}$ represent the volume per atom, bulk modulus, and its pressure derivative at the references pressure $P_{r}$. The values in Table II represent the results of the least-squares fits. The values of $\boldsymbol{P}_{r}$ were chosen thereby in the center of the stability region of each phase to obtain minimum uncertainties in $B_{r}$. For Se I, the values of $B_{r}$ and $B_{r}^{\prime}$ are found to be consistent with the earlier piston-cylinder data on hexagonal selenium. For the other phases, Table II presents the first sets of data for $B_{r}$ and $B_{r}^{\prime}$.
TABLE II. Values for the equation-of-state parameters $P_{r}$, $V_{r}, B_{r}$, and $B_{r}^{\prime}$ obtained from least-squares fits of the experimental data by Eq. (1).

\begin{tabular}{lclll}
\hline \hline Phases & $P_{r}(\mathrm{GPa})$ & $V_{r}\left(\AA^{3}\right)$ & $B_{r}(\mathrm{GPa})$ & \multicolumn{1}{c}{$B_{r}^{\prime}$} \\
\hline I & 7.7 & $21.56(13)$ & $48.1(20)$ & $4.33(4)$ \\
II & 20.4 & $14.50(8)$ & $63.7(36)$ & $2.53(8)$ \\
III & 35.2 & $12.94(5)$ & $263(14)$ & $5.2(2)$ \\
IV & 44.5 & $12.12(6)$ & $458(40)$ & $6.5(5)$ \\
\hline \hline
\end{tabular}

Since Se and Te show a close structural homology at low pressures, ${ }^{1,9,10}$ this homology has been studied for the low-pressure phase $\mathrm{SeI}$ and $\mathrm{TeI}$ also in a more extended pressure ranges by single-crystal $x$-ray diffraction ${ }^{38}$ and Raman scattering. ${ }^{40}$ These studies ${ }^{38,40}$ indicated already that deviations from simple scaling laws are observed as pressure approaches the first phase-transition pressure in either of these elements.

In addition, the present investigation shows remarkable differences at the structural phase transition between the phase I and II for Se and Te. The fact that the I-II-phase transition in Se occurs at a higher pressure with even a larger volume discontinuity (Fig. 2) than in $\mathrm{Te},{ }^{16}$ and the further observation that SeII, SeIII, and Se IV seem to have no structural resemblance with the corresponding phases of $\mathrm{Te}$, are considered as strong evidence for a breakdown of structural homology rules for these groupVI $A$ elements, at least in the present pressure range of investigations. However, if the $c / a$ value for SeIV reaches the value 0.612 , Se IV would change into a $b c c$ phase $\mathrm{SeV}$, which has been observed in $\mathrm{Te}$ as $\mathrm{Te} V$ at pressures in excess of $27 \mathrm{GPa}$ at room temperature. ${ }^{16}$ In the present pressure range of investigations the value of $c / a$ for the Se IV phase $(0.827)$ is found to be pressure independent. So it is difficult to estimate the transition pressure for a possible Se IV-Se V transition, keeping in mind that a bcc phase has been considered as a stable high-pressure phase for the group-VI $A$ elements. ${ }^{20}$ However, since there are no first-principles calculations available on the structural stability of the group-VI $A$ elements under high pressure, one cannot exclude that a structural homology will be resumed for these elements at very high pressure.

As mentioned earlier, the theoretical understanding of the high-pressure behavior of selenium is very limited $^{9-12,14,15}$ due to the lack of structural data. We hope that the present results will stimulate theorists to study the stability of the different phases of selenium and their electronic structure, in order to obtain ultimately a better understanding of the physics of group-VI $A$ elements.

We gratefully acknowledge the help of Olaf Schulte with the experimental work. This work has been funded in part by the German Minister for Research and Technology (BMFT) under the Contract No. 05-340-AxBO. One of us (G.P.) is grateful to the Alexander von Humbolt Foundation for financial support. 
*Permanent address: Materials Research Laboratory, Indian Institute of Science, Bangalore 560012, India.

'See The Physics of Selenium and Tellurium, edited by E. Gerlach and P. Groose (Springer-Verlag, Berlin, 1979).

${ }^{2}$ A. S. Balchan and H. G. Drickamer, J. Chem. Phys. 34, 1948 (1961).

${ }^{3}$ B. M. Riggleman and H. G. Drickamer, J. Chem. Phys. 37, 446 (1962).

${ }^{4}$ F. P. Bundy and K. J. Dunn, J. Chem. Phys. 71, 1550 (1979).

${ }^{5}$ G. Parthasarathy, K. J. Rao, and E. S. R. Gopal, Solid State Commun. 52, 867 (1984).

${ }^{6}$ J. Wittig, Phys. Rev. Lett. 15, 159 (1969).

${ }^{7}$ A. R. Moodenbaugh, C. T. Wu, and R. Viswanathan, Solid State Commun. 13, 1413 (1973).

${ }^{8}$ F. P. Bundy and K. J. Dunn, Phys. Rev. Lett. 44, 1623 (1980).

${ }^{9}$ J. D. Joannopoulos, M. Schüter, and M. L. Cohen, Phys. Rev. B 11, 2186 (1975).

${ }^{10}$ R. M. Martin, G. Lucovsky, and K. Helliwell, Phys. Rev. B 13, 1383 (1976).

${ }^{11}$ H. Wendel, R. M. Martin, and D. J. Chadi, Phys. Rev. Lett. 38, 656 (1977).

12 J. D. Joannopoulos, Th. Starkloff, and M. Kastner, Phys. Rev. Lett. 38, 660 (1977).

${ }^{13}$ G. Doerre and J. D. Joannopoulos, Phys. Rev. Lett. 43, 1040 (1979).

${ }^{14}$ J. von Boehm and H. M. Isomäki, Phys. Rev. B 24, 6945 (1981).

${ }^{15}$ H. M. Isomäki and J. von Boehm, Phys. Rev. B 35, 8019 (1987).

${ }^{16}$ G. Parthasarathy and W. B. Holzapfel, Phys. Rev. B 37, 8499 (1988).

${ }^{17}$ H. Olijnyk, S. K. Sikka, and W. B. Holzapfel, Phys. Lett. 103A, 137 (1984).

${ }^{18}$ Y. K. Vohra, K. E. Brister, S. Desgreniers, A. L. Ruoff, K. J. Chang, and M. L. Cohen, Phys. Rev. Lett. 56, 1944 (1986).

${ }^{19}$ S. J. Duclos, Y. K. Vohra, and A. L. Ruoff, Phys. Rev. Lett. 58, 775 (1987).

${ }^{20}$ L. G. Liu and W. A. Bassett, Elements, Oxides, and Silicates (Oxford Univ. Press, New York, 1986), pp. 24-91.
${ }^{21}$ D. R. McCann and L. Cartz, J. Chem. Phys. 56, 2552 (1972).

${ }^{22}$ H. K. Mao, G. Zou, and P. M. Bell, Carnegie Inst. Washington Yearb. 80, 283 (1980).

${ }^{23}$ S. N. Vaidya and G. C. Kennedy, J. Phys. Chem. Solids 33, 1377 (1972).

${ }^{24}$ A. K. Singh and G. C. Kennedy, J. Phys. Chem. Solids 35, 1545 (1974).

25J. Donohue, The Structure of the Elements (Wiley, New York, 1974), p. 370.

${ }^{26}$ G. K. Huber, K. Syassen, and W. B. Holzapfel, Phys. Rev. B 15, 5123 (1977).

${ }^{27}$ A. Jayaraman, Rev. Mod. Phys. 55, 65 (1983).

${ }^{28}$ R. A. Forman, G. J. Piermarini, J. D. Barnett, and S. Block, Science 176, 284 (1972).

${ }^{29}$ J. D. Barnett, S. Block, and G. J. Piermarini, Rev. Sci. Instrum. 44, 1 (1973).

${ }^{30}$ G. J. Piermarini, S. Block, J. D. Barnett, and R. A. Forman, J. Appl. Phys. 46, 2774 (1975).

${ }^{31}$ G. J. Piermarini and S. Block, Rev. Sci. Instrum. 46, 973 (1975).

${ }^{32}$ G. J. Piermarini (private communication).

${ }^{33}$ H. K. Mao, P. M. Bell, J. W. Shaner, and D. J. Steinberg, J. Appl. Phys. 49, 3276 (1978).

${ }^{34}$ K. Syassen and W. B. Holzapfel, Europhys. Conf. Abstr. 1A, 75 (1975)

${ }^{35}$ W. B. Holzapfel and W. May, in High Pressure Research in Geophysics, Advances in Earth and Planetary Sciences, Vol. 12, edited by S. Akimoto and M. M. Manghnani (Reidel, London, 1982), p. 73.

${ }^{36}$ W. B. Holzapfel, Rev. Phys. Appl. 19, 705 (1984).

${ }^{37}$ D. R. McCann, L. Cartz, R. E. Schmunck, and Y. D. Harker, J. Appl. Phys. 43, 1432 (1972).

${ }^{38}$ R. Keller, W. B. Holzapfel, and H. Schulz, Phys. Rev. B 16, 4404 (1977).

${ }^{39}$ K. Aoki, O. Shimomura, and S. Minomura, J. Phys. Soc. Jpn. 48, 551 (1980).

${ }^{40} \mathrm{~K}$. Aoki, O. Shimomura, S. Minomura, N. Koshizuka, and T. Tsushima, J. Phys. Soc. Jpn. 48, 906 (1980). 